\title{
Observing the NICEties of hypertension management
}

\author{
${ }^{1}$ EE Morrison, ${ }^{2} \mathrm{EJ}$ Turtle, ${ }^{3} \mathrm{DJ}$ Webb \\ ${ }^{1}$ Clinical Pharmacology and Therapeutics ST3, Royal Infirmary of Edinburgh; ${ }^{2}$ McKenzie Lecturer, University of Edinburgh; \\ ${ }^{3}$ Professor of Therapeutics and Clinical Pharmacology, University of Edinburgh, UK
}

This review is based on presentations given at the Symposium of the High Blood Pressure Foundation supported by RCPE on 23 November $201 \mathrm{I}$.

ABSTRACT National Institute for Health and Clinical Excellence guidance for the clinical management of hypertension, published last year, proposes a step change in UK clinical practice.' Although broadly helpful, there are some concerns about its implementation. Ambulatory blood pressure monitoring for diagnosis of hypertension, though logical, will place an additional financial burden on primary care at a time of austerity. Home blood pressure measurement may be a more practical option. Previous guidance recommended the used of thiazide diuretics as a first-line treatment option. ${ }^{2}$ Five years later, the new guidelines propose a major change, with an initial emphasis on the use of calcium channel blockers and angiotensin converting enzyme inhibitors/angiotensin receptor blockers, moving the use of thiazide-like diuretics to a third-line option. In addition, bendroflumethiazide, the mainstay of treatment in the UK over many years, has been replaced with chlortalidone, the starting doses of which are not readily available in this country. Cost-effectiveness analysis and a presumed risk of metabolic disorders has guided the rationale for these changes to the therapeutic algorithm, however this may not be robust. Importantly, unless there are special circumstances, reducing the blood pressure in hypertensive patients is more important than the means used to lower it. In future, it will be important to 'personalise' treatment more effectively and base management on lifetime risk.

KEYWORDS Hypertension, NICE, guidelines, research, blood pressure measurement, diuretics
Correspondence to DJ Webb, Pharmacology, Toxicology and Therapeutics

BHF Centre for Research

Excellence

University of Edinburgh, Queen's

Medical Research Institute

47 Little France Crescent

Edinburgh EHI6 4TJ,

Scotland, UK

tel. +44 (0) I3। 2429215

e-mail d.j.webb@ed.ac.uk

DECLARATION OF INTERESTS No conflicts of interest declared.

\section{INTRODUCTION}

One billion people worldwide are currently affected by hypertension, with the global burden set to rise to $>1.5$ billion by $2025 .{ }^{3}$ Hypertension is a major cause of death and disability in the developed and developing world and is associated with major adverse health outcomes including stroke, acute myocardial infarction (MI) and heart failure. ${ }^{4}$ Even small changes in blood pressure (BP) have a profound impact on population outcome. Indeed, a 2 millimetres of mercury $(\mathrm{mm} \mathrm{Hg})$ reduction in population systolic blood pressure should result in around a $7 \%$ reduction in population coronary risk and a $10 \%$ reduction in stroke risk. ${ }^{5}$

In the UK, hypertension affects one in four adults and is the main focus of $>10 \%$ of consultations in primary care. Prevalence within the over 75 age group currently stands at more than $60 \%$ in women and $70 \%$ in men. ${ }^{6}$ The financial burden to society is substantial: in 2004 , over $f \mid 5$ billion was spent on treatment and prevention of cardiovascular diseases,' though this figure is now falling because major classes of recommended drugs - angiotensinconverting enzyme (ACE) inhibitors, angiotensin receptor blockers (ARBs), calcium channel blockers (CCBs) and diuretics - are available in generic versions that cost only in the order of a few pounds $(E)$ for one year of treatment.

As a result of over 50 years of targeted research, there is a greater evidence base from clinical trials on the management of hypertension than for any other medical condition. Treatment of hypertension is one of the most cost-effective medical interventions, costing less than the vascular morbidity and mortality associated with no treatment. In hypertension, therefore, treatment costs less than doing nothing.

\section{NEW RECOMMENDATIONS}

Building on the early guidance published in $2006,{ }^{2}$ the National Institute for Health and Clinical Excellence (NICE) Guideline Development Group (GDG), in collaboration with the British Hypertension Society 
(BHS), published updated guidelines for the management of primary hypertension in adults (Table I) in August 201 I.' These new recommendations apply only to primary hypertension (the term 'essential' hypertension is no longer appropriate as we know hypertension to be a key risk factor for strokes and heart attacks at all ages and not 'essential' to maintain perfusion of atherosclerotic arteries). Hypertension in a diabetic population, accelerated hypertension, gestational hypertension and secondary hypertension remain the remit of other NICE guidelines and of specialist care. The new guidelines consist of 65 recommendations, of which 36 have been announced as new, reflecting the developing research base and prompting changes to the previous advice. Most of these changes have been identified as relatively straightforward to incorporate into clinical practice.

\section{TABLE I Key lessons}

\begin{tabular}{l} 
Hypertension is a major global cause of death and disability, \\
mainly through strokes and heart attacks \\
\hline Blood pressure reduction is key to reducing the risk, \\
regardless of which treatment is used \\
\hline Lifestyle advice and support is essential in all patients \\
Current recommendations for treatment: \\
Step I - ACE inhibitor or CCB \\
Step 2 -ACE inhibitor + CCB \\
Step $3-$ ACE inhibitor + CCB + TLD \\
Step $4-$ ACE inhibitor + CCB + TLD + spironolactone \\
\hline ABPM is recommended for confirmation of hypertension. \\
If patients are unable to undertake this, HBPM is a useful \\
alternative \\
\hline HBPM is cheap and reliable, whereas ABPM is currently \\
relatively costly \\
\hline Antihypertensive medications are, largely, cheap and the \\
treatment cost-effective \\
\hline Early referral to tertiary centers is important for patients \\
with resistant hypertension, or a clinical/biochemical \\
indication of a treatable cause \\
\hline ACE= angiotensin-converting enzyme; \\
CCB = calcium channel blocker; \\
TLD= thiazide-like diuretic; \\
ABPM= ambulatory blood pressure monitoring; \\
HBPM= home blood pressure monitoring \\
\hline
\end{tabular}

\section{Diagnosing hypertension}

Staging is used to assess risk and prompt the clinician to follow relevant management strategies (Table 2). Recognising the lifelong nature of treatment, the guidelines recommend the use of ambulatory BP monitoring (ABPM) to confirm hypertension in those identified at risk of stage one and stage two hypertension and standard clinic BP measurement (CBPM) used for severe hypertension. Ambulatory BP monitoring involves the average reading of twice hourly BP measurements, taken on a single day, during the patient's normal waking
TABLE 2 Diagnostic criteria for hypertension

Stage one hypertension: Clinic BP is $140 / 90 \mathrm{~mm} \mathrm{Hg}$ or higher and subsequent ABPM daytime average, or HBPM average, is $135 \mathrm{~mm} \mathrm{Hg}$ or higher

Stage two hypertension: Clinic BP is $160 / 100 \mathrm{~mm} \mathrm{Hg}$ or higher and subsequent ABPM daytime average, or HBPM average, is $150 / 95 \mathrm{~mm} \mathrm{Hg}$ or higher

Severe hypertension: Clinic systolic BP is $180 \mathrm{~mm} \mathrm{Hg}$ or higher or clinic diastolic BP is $110 \mathrm{~mm} \mathrm{Hg}$ or higher.

ABPM= ambulatory blood pressure monitoring;

HBPM= home blood pressure monitoring;

$\mathbf{B P}=$ blood pressure;

$\mathbf{m m ~ H g}=$ millimetres of mercury

hours. Home BP monitoring (HBPM) is the recommended alternative for patients unable or unwilling to use $A B P M$. It involves twice daily BP recording for at least four days, ideally seven, with each recording consisting of two consecutive measurements. The first day's measurements are discarded and an average value of all remaining ones are used.

\section{Threshold for treatment}

Patients under the age of 80 with stage one hypertension should be investigated for target organ damage (such as left ventricular hypertrophy, chronic kidney disease and hypertensive retinopathy), established cardiovascular disease and diabetes. If any of these conditions are present, or if the patient's ten-year cardiovascular risk is equivalent to $20 \%$ or greater, treatment should be offered. ${ }^{8}$ The guidelines recommend that all patients of any age with stage two or severe hypertension should progress immediately to treatment and lifestyle interventions should be addressed in everyone. The change in the definition of 'severe' hypertension is used to prompt the clinician to proceed immediately to treatment following CBPM rather than delaying treatment for ABPM. Referral to secondary care should be considered in the absence of target organ damage or significant cardiovascular risk if the patient is less than 40 years of age.

Five-yearly follow-up is recommended by NICE in patients where hypertension is not confirmed. If hypertension has been diagnosed and treatment started, once-yearly follow-up with CBPM has been suggested for re-evaluation of treatment, reassessment of targets and other relevant support.

\section{Antihypertensive treatment: initiation and titration of therapy}

Clinic BP that remains $\geq 140 / 90 \mathrm{~mm} \mathrm{Hg}$ for those under 80 years of age and $\geq 150 / 90 \mathrm{~mm} \mathrm{Hg}$ for those over 80 years of age is regarded as the threshold for progression to the next stage of treatment. Clinic BP monitoring 
remains the standard for assessing response to treatment. However, if a patient has a confirmed 'white coat' effect (a discrepancy of more than $20 / 10 \mathrm{~mm} \mathrm{Hg}$ between CBPM and ABPM/HBPM at the time of diagnosis), ABPM or HBPM should be considered as an adjunct. It is important to stress that patients with a stable, wellcontrolled BP established on bendroflumethiazide or other previously recommended medication should continue on this treatment. Generic medications should be offered, if appropriate, to minimise cost.

\section{Step I treatment}

People aged 55 years and over or Black people of African or Caribbean descent of any age should be started on a CCB. ${ }^{9}$ If a CCB is not suitable or if there is evidence of heart failure, a thiazide-like diuretic (TLD) should be offered. This refers to medications such as chlortalidone (previously chlorthalidone; $12.5 \mathrm{mg}-25.0 \mathrm{mg}$ once daily) or indapamide ( $1.5 \mathrm{mg}$ or $2.5 \mathrm{mg}$ once daily) in preference to a conventional thiazide-type diuretic (TTD) such as bendroflumethiazide or hydrochlorothiazide.

People aged less than 55 years should be started on an ACE inhibitor (such as ramipril or lisinopril). Side-effects include intractable cough, which occurs in around 10\% of patients. An ARB (such as candesartan or irbesartan) is a suitable alternative.

\section{Step 2 treatment}

Step 2 treatment now refers to the use of a $C C B$ in combination with either an $A C E$ inhibitor or an $A R B$ in the majority of patients. Again, if a CCB is not suitable because of intolerance, or if there is evidence of heart failure, a TLD should be considered. For Afro-Caribbeans, consider an ARB in preference to an ACE inhibitor, due to the increased risk of angioedema within this population. ${ }^{10}$ When considering combination therapy, NICE stresses the importance of avoiding the combination of beta blockers and TLDs, where possible, to reduce the risk of developing diabetes. Before considering proceeding to step 3 treatment, medication should be reviewed to ensure step 2 treatment is at optimal, or the best tolerated, doses.

\section{Step 3 treatment}

Triple therapy with an ACE inhibitor or an $A R B$ in combination with a CCB and a TLD is now recommended. At this stage, clinic BP that remains $\geq 140 / 90 \mathrm{~mm} \mathrm{Hg}$ on three drugs at optimal doses is regarded as treatment resistant hypertension. At this point expert advice should be sought before progressing to step 4 treatment.

\section{Step 4 treatment}

It is important at this stage to carefully explore any difficulties with adherence to therapy. At this step, further diuretic therapy is recommended. Low-dose spironolactone ( $25 \mathrm{mg}$ once daily) is preferred if blood potassium level is $\leq 4.5$ millimoles per litre $(\mathrm{mmol} / \mathrm{L})$, though caution should be exercised in those with renal impairment. It does not currently have a UK marketing authorisation for this indication so informed consent should be obtained. If blood potassium level is $\geq 4.5$ $\mathrm{mmol} / \mathrm{L}$, consider a higher dose of TLD. Blood sodium and potassium and renal function should be checked within one month and repeated as required thereafter. If further diuretic therapy is not tolerated, contraindicated or ineffective, consider an alpha blocker (such as doxazosin) or beta blocker (such as bisoprolol). If BP remains uncontrolled with optimal or maximum tolerated doses of four drugs, expert advice should be sought if it has not already been obtained.

\section{Education and adherence}

Patients who have not reached the threshold for diagnosis of hypertension should be followed up every five years. Patients who reach the criteria for treatment should be offered annual review to monitor BP, address targets, provide support and discuss lifestyle interventions. Clinicians can further help people to make informed choices by providing information about the benefits of treatment, directing people towards patient organisations and alerting them to possible sideeffects of particular drugs. Interventions to overcome non-adherence, if a specific issue is identified, include: recorded medicine-taking, patient monitoring, simplified dosing regimen or the use of a multi-compartment medicines system."

\section{Cost analysis}

The National Institute of Health and Clinical Excellence employed a complex cost analysis model, using Quality Adjusted Life Years (QALYs) based on the likelihood of progression to cardiovascular morbidity and mortality. Calcium channel blockers were found to be the most cost-effective intervention with regard to unstable angina, MI, stroke and mortality outcomes. Thiazide-like diuretics remain most cost-effective in the heart failure group and ACE inhibitors within a diabetic population. Calcium channel blockers are more cost-effective than TLDs in people with a higher risk of cardiovascular disease (CVD) and older age groups, though importantly this difference is not large. This change, in comparison to the $\mathbf{2 0 0 6}$ guidelines, reflects a considerable reduction in the incremental cost-effectiveness ratio (ICER) for CCBs, from $\ell \mid 2,250$ to $\ell I, 960$ per additional QALY, making the $C C B$ class much more cost-effective than previously.' This has led NICE to recommend the use of CCBs as first-line management for those aged $>55$ years in the hypertensive population. Furthermore, this low ICER means that treatment with CCBs are more cost-effective than no intervention, leading to the conclusion that 'treating hypertension is now cheaper than doing nothing'.' 


\section{CONTROVERSY OVER THE GUIDELINE}

\section{Clinic blood pressure measurement vs ambulatory blood pressure monitoring vs home blood pressure monitoring}

The routine use of $A B P M$ for the confirmation of hypertension is arguably the most controversial new recommendation in the NICE guidelines. It was found to be the most cost-effective option across a range of age subgroups in both men and women. The analysis assumed only people who are truly hypertensive (as diagnosed by $A B P M$ ) would receive benefit from treatment and assumed ABPM to be the reference standard (with 100\% sensitivity and specificity). These parameters were investigated by the GDG and variations in sensitivity and specificity values and cost-effectiveness were found, with HBPM becoming cost-effective when its sensitivity and specificity was set equal to that of ABPM. However, these assumptions can be challenged. Perhaps more concerning is the five-yearly follow-up of at-risk normotensive individuals that formed the backbone of the cost-effective analysis. It is likely that review will occur within a shorter time frame, making ABPM considerably less cost-effective.

For a UK general practice with a list of 10,000 patients, according to these guidelines there would be a projected 80-100 new patients presenting with hypertension each year. ${ }^{12}$ Five or six ABPM devices, costing approximately $£ I, 000$ each, would be required to diagnose hypertension within this group, at a cost of $£|0,000-£| 2,000$ per practice for training and resources. ${ }^{12} \mathrm{~A}$ major national financial undertaking will therefore be required in order to deliver ABPM widely. Although an ongoing national procurement scheme might drive down the cost of ABPM, the initial outlay is considerable. Extrapolating data from the cost analysis, NICE cites a potential national saving of $£ 10$ million, five years after national implementation.' ${ }^{2}$ Alternatively, a cheap BHS-validated device can be bought locally for as little as $£ \mid 5$ for HBPM. Given the current economic climate and the financial pressures on the National Health Service the additional setup cost of ABPM is a substantial investment and the savings will be made in secondary care while the costs are incurred at the primary care level. There are also still issues to resolve: there are no treatment trials randomised on the basis of ABPM and there is a lack of robust evidence comparing HBPM to ABPM, clearly a very important area for new research. Although HBPM is not currently recommended, if $A B P M$ is thought to add value over clinic measurements, then HBPM must surely be explored as the way forward.

According to NICE, ABPM results in fewer people being incorrectly diagnosed as having hypertension than using CBPM alone (which is linked to 'white coat syndrome'). The use of ABPM may therefore reduce consultation times, the cost of unnecessary drugs and the psychological and practical impacts (such as on the rising cost of insurance) that this diagnosis can have. ${ }^{13} \mathrm{~A}$ diagnosis of white coat hypertension (WCHT) however cannot be regarded, beyond reasonable doubt, as benign. Although the clinical importance of WCHT has been investigated in many studies, the benefits of treatment on end-organ damage and long-term outcome remain unclear. An increase in stroke and overall cardiovascular risk, compared with normotensive controls, was evident in a ten-year follow-up study and overall mortality of patients with WCHT is markedly higher than that of normotensive individuals, though lower than that of patients with sustained hypertension. . $^{14,15}$

Another issue raised in the new guidelines is the current inability to reliably monitor BP using electronic devices, either in the clinic or at home, in patients with atrial fibrillation. It would be invaluable if this difficulty could be overcome. The prevalence of atrial fibrillation increases in older age, affecting almost one in ten people aged 80 years or older, representing a significant proportion of our target population. ${ }^{16}$

\section{Monitoring}

Evidence for the use of HBPM/ABPM versus CBPM is not yet robust enough in randomised controlled trials (RCT) and meta-analysis to support its use for monitoring hypertension and response to treatment in patients already established on antihypertensive medication. The guidelines therefore concluded that CBPM should remain the standard for assessing response to treatment. This has led to the unusual situation of different methods being recommended for diagnosis (ABPM) and monitoring (CBPM) of disease progression. Expert opinion would suggest the use of ABPM (or HBPM) for both, with investigation and assessment a focus for future research.

\section{Pharmacology}

Although the body of evidence for the use of CCBs and TLDs has remained unchanged since 2006, the costeffectiveness analysis and the reduced CCB ICER has led to the recommendation of CCBs as the first-line therapy in the $>55$-year-old group. The analysis suggests that the cost-effectiveness of drug classes is dependent on their relative effects on the prevention of diabetes and heart failure. The model predicts that for people at low to intermediate risk of heart failure, CCBs are the most cost-effective option (and they reduce BP variability) and for people at high risk of heart failure, TLDs are the most cost-effective alternative, provided that they do not also have a high risk of diabetes. However, it would be premature to use this as an argument for the use of $\mathrm{CCBs}$, given that there is no prospective outcome data favouring a reduction of BP variability. 
'Low dose' bendroflumethiazide is currently the most commonly prescribed antihypertensive medication in Scotland and the NICE recommendations would therefore constitute a major change in prescribing practice. ${ }^{17}$ The shifting of TLDs to third-line therapy is largely due to concern regarding the risk of new onset diabetes. The body of evidence that the small average increase in glucose caused by TLDs translates into a significant change in cardiovascular outcomes is however not robust. ${ }^{18}$ Indeed, the increase may well also be reversible after starting alternative therapy. Furthermore, due to concerns regarding electrolyte disturbance, including hypokalaemia, the usual dose of bendroflumethiazide $(2.5 \mathrm{mg})$ is only a quarter of that used to show benefit for cardiovascular outcomes in clinical trials. The MRC trial demonstrated a significant reduction in stroke events using bendroflumethiazide against placebo and beta blockers, but only at the $10 \mathrm{mg}$ dose. ${ }^{19}$ Unfortunately, a widely cited study which apparently demonstrated no significant difference between bendroflumethiazide dose and BP reduction was underpowered and should not have formed the basis for current practice. ${ }^{20}$ Indeed, there is limited evidence to support the use of bendroflumethiazide $2.5 \mathrm{mg}$ daily and it may well be insufficient to provide benefit. Such evidence was available well before the 2006 NICE guidelines, but only now being acted on. As identified by NICE, TLDs could be considered as first-line therapy in those with evidence of, or at higher risk of, heart failure but it remains unclear what defines this 'at risk' population.

Choosing between thiazide diuretics remains difficult. Concurrent studies have shown trends favouring chlortalidone as a more effective BP lowering agent than hydrochlorothiazide, but statistical significance has not been consistently demonstrated. ${ }^{21}$ The number of confounding variables - including dosages and use of other antihypertensives - makes robust analysis difficult. The most recent evidence comparing hydrochlorothiazide and chlortalidone for BP management was published in 2006.22 Using ABPM, chlortalidone was shown to reduce $\mathrm{BP}$ more than hydrochlorothiazide only during the night time hours, with no difference during the day and a trend only in 24-hour mean systolic $\mathrm{BP}$ reduction. Regarding cardiovascular outcomes, a recent retrospective observational cohort study showed that people on chlortalidone had significantly fewer cardiovascular events ( $\mathrm{Ml}$, coronary artery bypass, coronary artery disease and peripheral artery disease).$^{23}$ These data were extrapolated from the Multiple Risk Factor Intervention Trial (MRFIT) study, which had already suggested chlortalidone might offer greater benefit. ${ }^{24}$ However, the information on deaths in this analysis was not fully described and a number of confounders exist. A recent meta-analysis of dose response relationships of thiazide diuretics for systolic BP demonstrated a log-linear relationship with a potency series: bendroflumethiazide>chlortalidone>hydrochlorothiazide. Similar changes in serum potassium and uric acid, if equivalent doses are used, suggest no obvious difference in efficacy between the three. ${ }^{25}$ Prospective randomised studies are needed to confirm the effects on clinical outcomes.

Chlortalidone use has however been recommended by NICE, starting at $12.5 \mathrm{mg}$ daily, but the drug is only available in the UK as a $50 \mathrm{mg}$ tablet. Although the tablet can be halved (with difficulty), $12.5 \mathrm{mg}$ dosing is hard to achieve. One possibility is alternate-day dosing with 25 $\mathrm{mg}$, as chlortalidone has a long half-life of 45 to 60 hours, but adherence may be reduced. It must be hoped that a generic version of the full range of doses (12.5 mg, 25 $\mathrm{mg}$ and $50 \mathrm{mg}$ ) will soon be made available.

\section{Combination therapy}

There is a paucity of evidence regarding combination therapy and the effectiveness of treatment but this is being addressed by ongoing research. Existing evidence would suggest that starting with combination therapy may be superior to starting with one drug and adding a second agent at a later date. ${ }^{26,27}$ Furthermore, recent evidence would suggest single pill combination therapy is more effective at hypertension control than free combination during the first treatment year of hypertensive patients. ${ }^{28}$

\section{Elderly population}

Few studies have evaluated hypertension in the elderly and studies that included patients $>80$ years of age enrolled too few to draw firm conclusions. Meta-analysis of available RCTs would suggest treating hypertension in very old patients reduces stroke and heart failure but has no effect on total mortality, with meta-regression suggesting that a reduction in mortality was achieved in trials with the least BP reductions and the lowest intensity of therapy. ${ }^{29}$ Although the Hypertension in the Very Elderly Trial (HYVET) study did have adequate power to evaluate the very elderly, this study was stopped early because of lower mortality in the treatment group, so unanswered questions remain regarding optimal BP targets. ${ }^{30}$ The association of antihypertensive treatment with reduced rates of cognitive decline or dementia has also been controversial. Meta-analysis of 14 longitudinal studies showed that antihypertensive medication use could decrease the risk of developing dementia (excluding Alzheimer's disease) and the associated cognitive decline. ${ }^{31}$ Stopping antihypertensive therapy must, therefore, be a judgement based on the patient reaching a level of frailty where statistical benefit from treatment no longer outweighs other factors. 


\section{Patients under 40 years old}

There is genuine uncertainty about whether to treat people under 40 years of age with stage one hypertension with no clinical evidence of target organ damage. Secondary causes of hypertension, rather than primary, are more common within this group and should be excluded. It is unclear which patients in this group will progress and which will develop significant cardiovascular morbidity and mortality. Answering these questions would require outcome trials too large, too long and too costly to be feasible. In a recent Swedish nationwide study of more than 1.2 million young men with up to 37 years of follow-up, the relationship of diastolic BP to mortality was found to be positive, with an apparent risk threshold of a BP around $90 \mathrm{~mm} \mathrm{Hg.}{ }^{32}$ While undertaking the required research in this important area, we must reflect on the impact or penalties that a diagnosis of hypertension on a young adult will incur, notably with regard to the cost of health insurance. ${ }^{13}$ Current recommendations continue to use the ten-year cardiovascular risk measurement though NICE acknowledge this approach underestimates the risk to those under the age of 40 because short-term risk assessment is powerfully influenced by age. Nevertheless, the lifetime risk associated with untreated stage one hypertension in this age group could be substantial. Lifetime risk assessments may be a better way to inform treatment decisions and evaluate the cost-effectiveness of earlier intervention with pharmacological therapy.

\section{ONGOING ISSUES}

The percentage of people with hypertension in the UK attaining the BP target of $<140 / 90 \mathrm{~mm} \mathrm{Hg}$ is low. In England, only $29 \%$ of the hypertensive population meet this target and it is therefore important to reflect on the impact of guidance on clinical practice. The Quality and Outcomes Framework (QOF) addresses this issue but the pay for performance targets for high quality care has had little effect on processes or on hypertension-related clinical outcomes in the UK. ${ }^{33}$ The BHS has recommended that the QOF standard should be set at $90 \%$ of the hypertensive population reaching a target $\mathrm{BP}$ of $<150 / 90$ $\mathrm{mm} \mathrm{Hg}$ and $50 \%<140 / 90 \mathrm{~mm} \mathrm{Hg}$. An ongoing UK pilot study will determine how effective the implementation of these targets will be and the related cost.

Large multicentre prospective trials are required to answer fundamental questions. The BHS's British Heart Foundation funded Prevention and Treatment of Hypertension With Algorithm-based Therapy (PATHWAY) study consists of three trials to address some of the unanswered questions regarding hypertension management. Similar to the aliskirin and the $C C B$ amlodipine combination as an initial treatment strategy for hypertension control (ACCELERATE) trial, PATHWAY I uses a different pair of drug classes (ARB and TLD) instead of a renin inhibitor and $C C B$ to address whether combination treatment is more effective than one drug followed by the addition of another. PATHWAY 2 addresses resistant hypertension, asking whether spironolactone is the best fourth-line drug and whether exceptions can be predicted by measuring plasma renin. PATHWAY 3 compares available single and combination diuretic formulations, hydrochlorothiazide and amiloride, for both hypertensive efficacy and incidence of glucose intolerance.

Academic debate will and should continue around the latest NICE recommendations for the management of hypertension. It is clear that no single treatment is right for all patients, but with the wide range of treatments available, it should be possible to find a combination that suits most. Treatment of hypertension is one of the most cost-effective medical interventions in healthcare and it is crucial that we have access to the best possible evidence-based guidance on its management, provided in a way that ensures good uptake of the advice.

\section{REFERENCES}

I National Institute for Health and Clinical Excellence. Hypertension: the clinical management of primary hypertension in adults. CGI 27 [Internet]. London: National Clinical Guideline Centre; 201 I [cited 2012 June 17]. Available from: http://www.nice.org.uk/nicemedia/ live//3561/56007/56007.pdf

2 National Institute for Health and Clinical Excellence. Hypertension: management of hypertension in adults in primary care. CG34 [Internet]. London: NICE; 2006 [cited 2012 July 26].Available from: http://www.nice.org.uk/nicemedia/pdf/CG034NICEguideline.pdf

3 Kearney PM, Whelton M, Reynolds $\mathrm{K}$ et al. Global burden of hypertension: analysis of worldwide data. Lancet 2005; 365:2 I 7-23. http://dx.doi.org/doi:I0.1016/S0|40-6736(05) I774I-I

4 Lopez AD, Mathers CD, Ezzati M et al. Global and regional burden of disease and risk factors, 200 I: systematic analysis of population health data. Lancet 2006; 367:I747-57. http://dx.doi.org//0.1016/ SOI40-6736(06)68770-9

5 Lewington S, Clarke R, Qizilbash N et al. Age specific relevance of usual blood pressure to vascular mortality: a meta-analysis of individual data for one million adults in 61 prospective studies. Lancet 2002; 360:1903-13. http://dx.doi.org/10.1016/S0I406736(02)II9II-8

6 Craig R, Mindell J (editors). Health survey for England 2006. Volume I: Cardiovascular disease and risk factors in adults [Internet]. Leeds: The Information Centre; 2008 [cited 2012 June I7].Available from: http://www.ic.nhs.uk/webfiles/publications/HSE06/HSE\%2006\%20 report\%20VOL\%20I\%20v2.pdf

7 Luengo-Fernández R, Leal J, Gray $\mathrm{A}$ et al. Cost of cardiovascular diseases in the United Kingdom. Heart 2006; 92:I384-9. http:// dx.doi.org//0.1 I36/hrt.2005.072/73

8 National Institute for Health and Clinical Excellence. Lipid modification. CG67 [Internet]. London: The National Collaborating Centre for Primary Care; 2008 [cited 2012 June 17].Available from: http://www.nice.org.uk/nicemedia/live/I I 982/40742/40742.pdt 
9 Leenen FH, Nwachuku CE, Black HR et al. Clinical events in highrisk hypertensive patients randomly assigned to calcium channel blocker versus angiotensin-converting enzyme inhibitor in the antihypertensive and lipid-lowering treatment to prevent heart attack trial. Hypertension 2006; 48:374-84. http://dx.doi. org/I0.1 I6I/0I.HYP.000023 I662.77359.de

I0 Piller LB, Ford CE, Davis BR et al. Incidence and predictors of angioedema in elderly hypertensive patients at high risk for cardiovascular disease: a report from the Antihypertensive and Lipid Lowering Treatment to Prevent Heart Attack Trial (ALLHAT) J Clinical Hypertens (Greenwich) 2006; 8:649-6. http://dx.doi.org/ I0.I I I I/ j. I524-6I75.2006.05689.x

II National Institute for Health and Clinical Excellence. Medicine adherence. CH76 [Internet].London: The National Collaborating Centre for Primary Care; 2009 [cited 2012 June 17].Available from: http://www.nice.org.uk/nicemedia/live/ I I766/4297I/4297I.pdf

12 National Institute for Research Excellence. Hypertension: costing report. Implementing NICE guidance. CGI27 [Internet]. London: NICE; 201 I [cited 2012 June I7]. Available from: http://www.nice. org.uk/nicemedia/live//356 I/560| 6/560 I6.pdf

13 Johnston ME, Gibson ES, Terry CW et al. Effects of labelling on income, work and social function among hypertensive employees.J Chron Dis 1984; 37:417-23. http://dx.doi.org//0.1016/002I968I(84)90025-0

14 Gustavsen PH, Høegholm A, Bang LE et al.White coat hypertension is a cardiovascular risk factor: a 10-year follow-up study. J Hum Hypertens 2003; I 7:8 I I-7.http://dx.doi.org/I0.1038/sj.jhh. I00I643

I5 Sega R, Facchetti R, Bombelli $M$ et al. Prognostic value of ambulatory and home blood pressures compared with office blood pressure in the general population: follow-up results from the Pressioni Arteriose Monitorate e Loro Associazioni (PAMELA) study. Circulation 2005; I I I:I777-83. http://dx.doi.org//0.1 I6I/0I. CIR.0000I60923.04524.5B

16 Go AS, Hylek EM, Phillips KA et al. Prevalence of diagnosed atrial fibrillation in adults: national implications for rhythm management and stroke prevention: the AnTicoagulation and Risk Factors in Atrial Fibrillation (ATRIA) Study. JAMA 200I; 285:2370-5. http:// dx.doi.org/I0.100I/jama.285.18.2370

17 ISD Scotland. Prescribing and medicines: prescription cost analysis financial year 2010/20 I I [Internet]. Edinburgh: ISD Scotland; 2012 [cited 2012 June 17]. Available from: http://www.isdscotland.org/Health-Topics/ Prescribing-and-Medicines/Publications/data-tables.asp

I8 Sofat R, Casas JP, Grosso AM et al. Could NICE guidance on the choice of blood pressure lowering be simplified? BMJ 2012; 344:d8078. http://dx.doi.org/ I0.I I36/bmj.d8078

19 MRC trial of treatment of mild hypertension: principal results. Medical Research Council Working Party. Br Med J (Clin Res Ed) 1985; 291:97-104. http://dx.doi.org//0.1 I36/bmj.29I.6488.97

20 Carlsen JE, Køber L, Torp-Pedersen C et al.Relation between dose of bendrofluazide, antihypertensive effect, and adverse biochemical effects. BMJ 1990; 300:975-8. http://dx.doi.org//0.II36/ bmj.300.6730.975
2I Neff KM, Nawarskas J. Hydrochlorothiazide versus chlorthalidone in the management of hypertension. Cardiol Rev 2010; 18:5I-6. http://dx.doi.org//0.1097/CRD.0b013e3 I8I c6 Ib52

22 Ernst ME, Carter BL, Goerdt CJ et al. Comparative antihypertensive effects of hydrochlorothiazide and chlorthalidone on ambulatory and office blood pressure. Hypertension 2006; 47:352-8. http:// dx.doi.org/I0.I I6I/0I.HYP.0000203309.07I40.d3

23 Dorsch MP, Gillespie BW, Erickson SR et al. Chlorthalidone reduces cardiovascular events compared with hydrochlorothiazide: a retrospective cohort analysis. Hypertension 2011; 57:689-94. http://dx.doi.org/I0.1 I6I/HYPERTENSIONAHA.I I0.16I505

24 Mortality after $10 \mathrm{I} / 2$ years for hypertensive participants in the Multiple Risk Factor Intervention Trial. Circulation 1990; 82:161628. http://dx.doi.org/I0.II6I/0I.CIR.82.5.1616

25 Peterzan MA, Hardy R, Chaturvedi $\mathrm{N}$ et al. Meta-analysis of doseresponse relationships for hydrochlorthiazide, chlorthalidone and bendroflumethiazide on blood pressure, serum potassium and urate. Hypertension 2012; 59:I 104-9. http://dx.doi.org//0.II6I/ HYPERTENSIONAHA.III.I90637

26 Brown MJ, Mclnnes GT, Papst CC et al. Aliskiren and the calcium channel blocker amlodipine combination as an initial treatment for hypertension control (ACCELERATE): a randomised, parallel-group trial. Lancet 20II; 377:312-20. http://dx.doi.org/I0.I0I6/SOI406736(I0)62003-X

27 Wald DS, Law M, Morris JK et al. Combination therapy vs monotherapy in reducing blood pressure: meta-analysis on $1 \mathrm{I}, 000$ participants from 42 trials. Am J Med 2009; 122:290-300. http:// dx.doi.org/I0.1016/j.amjmed.2008.09.038

28 Egan BM, Bandyopadhyay D, Shaftman SR et al. Initial monotherapy and combination therapy and hypertension control the first year. Hypertension 2012; 59:II24-3I. http://dx.doi.org/l0.II6I/ HYPERTENSIONAHA.II2.194I67

29 Bejan-Angoulvant T, Saadatian-Elahi M,Wright JM et al. Treatment of hypertension in patients 80 years and older: the lower the better? A meta-analysis of randomized controlled trials.J Hypertens 20I0;28: I 366-72.http://dx.doi.org/ I0.1097/HJH.0b0I3e328339f9c5

30 Beckett NS, Peters R, Fletcher AE et al. Treatment of hypertension in patients 80 years of age or older. N Engl J Med 2008; 358: I88798. http://dx.doi.org/ I0.1056/NEJMoa0801369

3I Chang-Quan H, Hui W, Chao-Min W et al. The association of antihypertensive medication use with risk of cognitive decline and dementia: a meta-analysis of longitudinal studies. Int J Clin Pract 201 I;65:1295-1305. http://dx.doi.org/I0.IIII/j.1742-I24I.20II.028I0.x

32 Sundström J, Neovius M, Tynelius $P$ et al. Association of blood pressure in late adolescence with subsequent mortality: cohort study of Swedish male conscripts. BMJ 20I I; 342:d643. http:// dx.doi.org/l 0.I I36/bmj.d643

33 Serumaga B, Ross-Degnan D, Avery AJ et al. Effect of pay for performance on the management and outcomes of hypertension in the United Kingdom: interrupted time series study. BMJ 20I I; 342:d I08. http://dx.doi.org/I0.I I36/bmj.dI 08 